\title{
EVALUATION OF THE SIGN DETECTOR FOR DCT DOMAIN WATERMARK DETECTION
}

\author{
FABING DUAN \\ Institute of Complexity Science \\ Department of Automation Engineering \\ Qingdao University, Qingdao 266071, P. R. China \\ fabing1974@yahoo.com.cn \\ DEREK ABBOTT \\ Centre for Biomedical Engineering (CBME) and \\ School of Electrical \& Electronic Engineering \\ The University of Adelaide \\ SA 5005, Australia \\ dabbott@eleceng.adelaide.edu.au \\ FRANÇOIS CHAPEAU-BLONDEAU \\ Laboratoire d'Ingénierie des Systèmes Automatisés (LISA) \\ Université d'Angers \\ 62 avenue Notre Dame du Lac, 49000 Angers, France \\ chapeau@univ-angers.fr
}

Received 28 March 2011

Accepted 13 June 2011

Communicated by Igor Goychuk

Reordering by the rule of decreased absolute amplitudes, the discrete cosine transformation (DCT) coefficients of an image are approximately modeled as dichotomous noise. Based on this assumption, it is interesting to note that the classical multiplicative embedding method can be transformed into an additive embedding rule, which accords with the signal processing problem of detecting a known weak signal in additive non-Gaussian noise. Then, following the generalized Neyman-Pearson lemma, a locally optimum detector, named the sign detector, is introduced to distinguish the correct watermark from the wrong ones. The statistical characteristics of this nonlinear sign detector are analytically investigated in detail. Extensive experimental results demonstrate the robustness of watermark against some common attacks, e.g., JPEG compression, cropping, filtering, additive Gaussian noise, dithering, and also verify the robust performance of the nonlinear sign detector for watermark detection.

Keywords: Nonlinear sign detector; Neyman-Pearson lemma; watermark detection; robustness. 


\section{Introduction}

Digital image watermarking, as a tool for copyright protection of images, has attracted significant attention [1-12]. A digital watermark contains information about the copyright owner, the authorized consumer or other information needed to be embedded, while the image acts as a host to which digital watermarks are adhered to [1-13]. So far, there are mainly two main proposed watermarking techniques, i.e., embedding the watermark in the spatial domain $[1,5-7,12,13]$ or in the transformed domain $[1,6,11,16,18,26,27]$ of a host image. It has been shown that the discrete cosine transformation (DCT) coefficients of images have interesting properties that can be exploited for watermark detection [14-29].

A well-known DCT-domain watermarking technique was proposed in [16], and developed in [18]. The watermark consists of a pseudo-random sequence with a normal distribution, and is embedded to a selected set of DCT coefficients of a host image $[16,18]$. In the course of watermark detection, the optimal correlation-based detector is adopted as a linear detector with the assumption of DCT coefficients following a Gaussian distribution $[16,18]$.

Progressively, many possible distributions of the DCT coefficients have been proposed, including Weibull [17], Cauchy [19, 20], generalized Gaussian [22-27], Laplacian $[24,25]$, etc. For these non-Gaussian probability density functions (pdf), a locally optimum detector is usually derived for watermark detection $[17,19,20$, 26-29]. Especially, Barni et al. noted that the proposed watermark embedding rule in $[16,18]$ does not obey an additive rule, and presented a new decoder for the optimum recovery of watermarks [17].

In this paper, we consider the detection of a digital watermark embedded in DCT coefficients of an image. The embedding rule mainly follows that proposed by Barni [18] and Stanković [28]. Firstly, the DCT coefficients of an image are reordered by their decreased absolute values, and a normal random variable, that is, watermark, is embedded into a selected segment of DCT coefficients. Next, we assume the selected segment of DCT coefficients as dichotomous noise for the watermark signal. Interestingly, the classical multiplicative embedding rule in $[16,18]$ can be transformed into an additive relationship between the DCT coefficients (noise) and the watermark (signal), which can be viewed as the signal detection problem of a known weak signal in additive non-Gaussian noise. This kind of transformation of the embedding rule is an important distinction from previous related studies $[16-18,28]$.

Theoretical analyses and experimental results also demonstrate the assumptions of the dichotomous pdf model of DCT coefficients and the Gaussian mixture pdf of the marked DCT coefficients by watermark. In order to discern the correct watermark from the wrong ones, a locally optimum detector, named the sign detector, is introduced by following the generalized Neyman-Pearson lemma. The theoretical statistical features of this nonlinear sign detector are discussed in detail, and the corresponding decision threshold is also deduced theoretically for a given false 
alarm probability. Experiments of watermark under no attack are performed, and demonstrate the theoretical analysis of the sign detector well. Finally, extensive robustness experiments for the watermark detection have been carried out to evaluate the effectiveness of the nonlinear sign detector under various common attacks, and the corresponding experimental results further validate the robustness of the proposed watermarking scheme.

This paper is organized as follows. The watermarking scheme will be briefly described in Sec. 2. Based on the assumption of a non-Gaussian pdf model of DCT coefficients of an image, a locally optimum detector is derived for the watermark detection. The statistical characteristics of this locally optimum detector are detailedly discussed. Main experimental results will be presented in Sec. 3 . Finally, we will discuss some further investigations, and draw our conclusions in Sec. 4.

\section{Watermarking Scheme}

In this section, we briefly introduce the considered watermarking scheme proposed by Barni [18] and Stanković [28], as follows. Firstly, the signal W= $\left\{w_{1}, w_{2}, \ldots, w_{K}\right\}$ generated by a pseudo-random normal vector is treated as the watermark [16-18], and the watermark length is $K$. Then, the $N \times N$ DCT coefficients for a $N \times N$ gray image $\mathbf{I}$ are computed, and then reordered into a one dimensional decreasing sequence [28] according to

$$
\mathbf{T}=\left\{t_{i}|| t_{i+1}|\leq| t_{i} \mid, i=1,2, \ldots, N^{2}\right\} .
$$

An illustrative segment of $\mathbf{T}$ taken from the standard test image "Lena" is shown in Fig. 1(a). Similar results have been obtained on other test images of "Boat", "Bridge", "Goldhill" and "Couple". This kind of reordering was put forward by Stanković and coworkers [28], which is different from the zig-zag scan reordering of Barni et al. [18]. Here, $\mathbf{T}$ is regarded as the background noise for the watermark signal W.

In order to obtain the perceptual invisibility of the watermark for the marked image and the robust watermark against common signal processing operations, the first $L$ reordered DCT coefficients of $\mathbf{T}$ in the low frequency domain usually avoid being marked [16-18]. The position $L$ is usually selected from the media frequency domain and the watermark $\mathbf{W}$ is robust to the high pass filtering [16-18]. From the $(L+1)$ th reordered DCT coefficients of the sequence $\mathbf{T}$, the watermark $\mathbf{W}$ is inserted into $\mathbf{T}$ according to the following rule [16-18]

$$
t_{L+k}^{\prime}=t_{L+k}+\alpha\left|t_{L+k}\right| w_{k}
$$

where $k=1,2, \ldots, K$, and $\alpha(0<\alpha \ll 1)$ is the scale parameter controlling the watermark strength [18-22]. The marked DCT coefficients $\mathbf{T}^{\prime}=$ $\left\{t_{L+1}^{\prime}, t_{L+2}^{\prime}, \ldots, t_{L+K}^{\prime}\right\}$ are formed. 


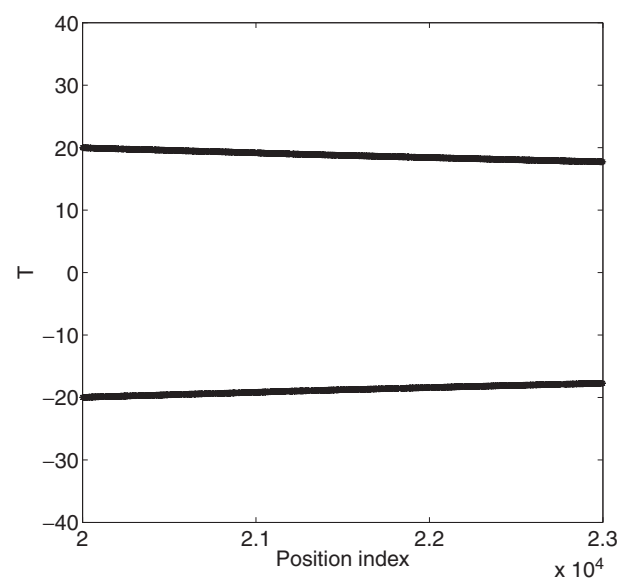

(a)

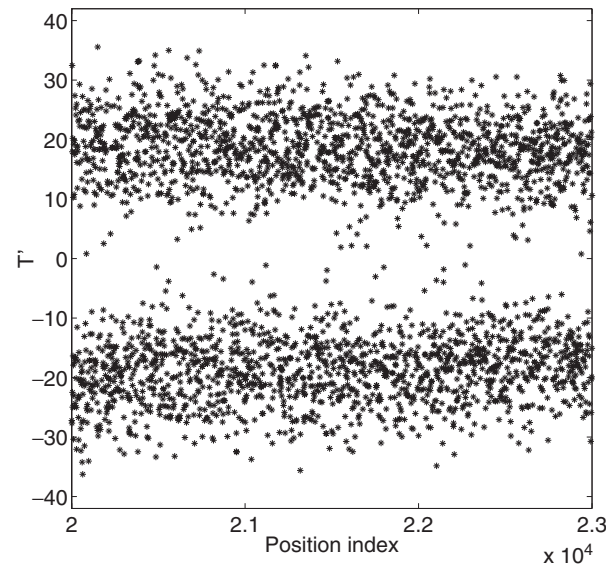

(b)

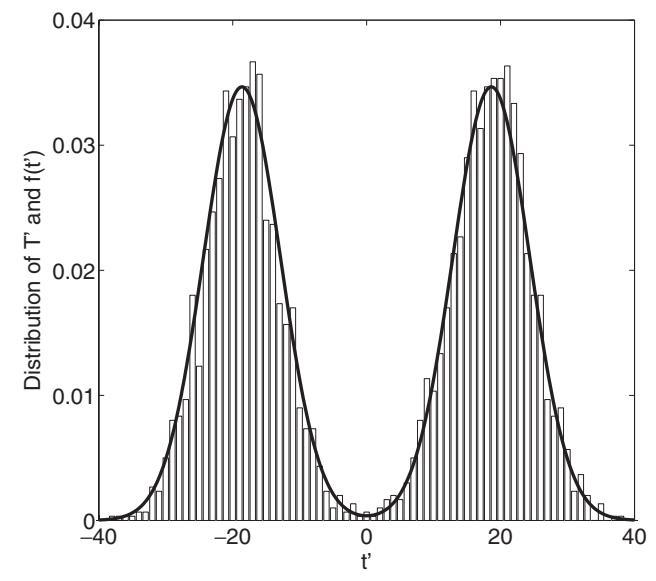

(c)

Fig. 1. A segment of (a) DCT coefficients $\mathbf{T}$ reordered by the rule of Eq. (1) with the length $K=3000$, and (b) the corresponding marked DCT coefficients $\mathbf{T}^{\prime}$ by the watermark $\mathbf{W}$ according to the rule of Eq. (2). Here, the referred test image is "Lena". The inserted position $L=20,000$, the watermark length $K=3000$ and the scale parameter $\alpha=0.3$. (c) Statistical distribution of marked DCT coefficients $\mathbf{T}^{\prime}$ of (b), with an approximated Gaussian mixture pdf $f\left(t^{\prime}\right)$ (solid line) of Eq. (9). Here, for the generated watermark with seed 100 by MATLAB software, the estimated parameters $\hat{\mu}=18.7801$ and $\hat{\theta}=5.6616$ computed by Eqs. (A.8) and (A.9) (see Appendix A).

Next, the $\mathbf{T}^{\prime}$ coefficients are put back to their original $K$ positions indicated by the original image $\mathbf{I}$. Finally, the $N \times N$ marked DCT coefficients are performed by the inverse DCT transformation, resulting in the marked image $\mathbf{I}^{\prime}$. Note that the original $K$ positions of DCT coefficients $\mathbf{T}$ in the host image $\mathbf{I}$ and the watermark W must be attainable for the following watermark recovery process, whereby the 
original $K$ positions of DCT coefficients can be viewed as a set of secret keys for watermark detection. This is the DCT-domain watermark embedding process proposed by $[18,28]$. In order to evaluate the degradation of the marked image $\mathbf{I}^{\prime}$, a commonly used measure is the PSNR defined as [16-18]

$$
\mathrm{PSNR}=10 \log _{10}\left(\frac{255^{2}}{\mathrm{MSE}}\right)
$$

where MSE indicates the mean square error of pixel values between the marked image $\mathbf{I}^{\prime}$ and the original one $\mathbf{I}$.

With no attacks on the marked image $\mathbf{I}^{\prime}$, the inverse process of the abovementioned watermark embedding algorithm still yields the sequence $\mathbf{T}^{\prime}$, with the stored $K$ positions of DCT coefficients in the host image $\mathbf{I}$. However, for a possibly attacked image $\mathbf{I}^{*}$, the inverse process of the above watermark embedding algorithm will give a sequence $\mathbf{T}^{*}=\left\{t_{L+1}^{*}, t_{L+2}^{*}, \ldots, t_{L+K}^{*}\right\}$ of DCT coefficients. Then, a watermark detector is operated for determining if the watermark $\mathbf{W}$ exists or if the watermark is the correct one that we embedded into images. This is the DCT-domain watermark recovering process [16-28].

\subsection{Probability density models of DCT coefficients}

For the watermark embedding algorithm of Eqs. (1)-(2), Stanković et al. [28] assumed the marked DCT coefficients $\mathbf{T}^{\prime}$ with a class of exponential pdf, and proposed the corresponding watermark detector. However, a mathematical difficulty for constructing the watermark detector is that the $1 / t_{L+k}^{\prime}$ term should avoid zeros in the denominator [28].

According to the ordering rule of Eq. (1), an example of the segment of DCT coefficients $\mathbf{T}$ with length $K=3000$ is shown in Fig. 1(a). It is seen that the absolute values of $\mathbf{T}$ do not change much (about in an interval of $[18,20])$, and there are 1554 positive values versus 1446 negative values in the length $K=3000$, in an approximately symmetrical way. Thus, in a certain length $K$ of the segment of DCT coefficients $\mathbf{T}$, we view the statistical characteristics of $t_{L+k}$ as a stationary stochastic process. We then assume that the DCT coefficients $t_{L+k}$ take two equiprobable values of $\pm \mu(\mu>0)$, and have a dichotomous pdf

$$
f_{T}(t)=\frac{1}{2} \delta(t-\mu)+\frac{1}{2} \delta(t+\mu)
$$

with a zero mean and a root mean square (RMS) of $\mu$. Here, the function of $\delta(t)$ in Eq. (4) is defined as the limit of a Gaussian pdf

$$
\delta(t)=\lim _{\epsilon \rightarrow 0} \frac{1}{\sqrt{2 \pi \epsilon^{2}}} \exp \left[-\frac{t^{2}}{2 \epsilon^{2}}\right]
$$


where $\epsilon^{2}$ is the variance of Gaussian pdf. Thus, Eq. (4) can be rewritten as

$$
f_{T}(t)=\lim _{\epsilon \rightarrow 0} \frac{1}{\sqrt{2 \pi \epsilon^{2}}} \exp [-\kappa(t \mid \mu, \epsilon)]
$$

with

$$
\kappa(t \mid \mu, \epsilon)=\frac{t^{2}+\mu^{2}}{2 \epsilon^{2}}-\ln \left[\cosh \left(\frac{\mu t}{\epsilon^{2}}\right)\right] .
$$

It is interesting to note that, on the assumption of the selected DCT coefficients of $\mathbf{T}$ with a dichotomous pdf of Eq. (4), the absolute value of $\mathbf{T}$ in the embedding rule of Eq. (2) becomes a constant $\mu$, i.e., $\left|t_{L+k}\right|=\mu$ for $k=1,2, \ldots, K$. Thus, the marked DCT coefficient $t_{L+k}^{\prime}$ becomes the sum of the selected DCT coefficient $t_{L+k}$ (noise components) and the scaled Gaussian watermark $\alpha\left|t_{L+k}\right| w_{k}=\alpha \mu w_{k}$ (signal components). This indicates that the embedding rule of Eq. (2) is directly converted into an additive formula as

$$
t_{L+k}^{\prime}=t_{L+k}+\theta w_{k}
$$

for $k=1,2, \ldots, K$ and $\theta=\alpha \mu$. Since $\mathbf{W}$ is a Gaussian random signal with zero mean, unity variance and its pdf $f_{W}(w)=\exp \left[-w^{2} / 2\right] / \sqrt{2 \pi}$, the pdf of marked DCT coefficients of $\mathbf{T}^{\prime}$ can be computed by

$$
f\left(t^{\prime}\right)=\int_{-\infty}^{\infty} f_{T}(t) f_{W}\left(t^{\prime}-t\right) d t=\frac{1}{\sqrt{2 \pi \theta^{2}}} \exp \left[-\kappa\left(t^{\prime} \mid \mu, \theta\right)\right],
$$

which is a Gaussian mixture pdf with the RMS of $\theta$ and two mean values of $\pm \mu$ for two corresponding peak points. For illustration purposes, the marked DCT coefficients $\mathbf{T}^{\prime}$, as shown in Fig. 1(b), are formed by embedding the watermark W into the segment of DCT coefficients $\mathbf{T}$, as illustrated in Fig. 1(a). It is seen in Fig. 1(b) that, due to the insertion of the watermark $\mathbf{W}$, the ordered DCT coefficients $\mathbf{T}$ are disordered as $\mathbf{T}^{\prime}$.

In practice, for a given data of marked DCT coefficients $\mathbf{T}^{\prime}$, we only know that $\mathbf{T}^{\prime}$ might contain the watermark signal $\mathbf{W}$, but the parameters $\mu$ and $\theta$ in Eq. (9) are unknown. Here, we assume that DCT coefficients $\mathbf{T}^{\prime}$ are independent and identically distributed. In Appendix A, according to the Gaussian mixture pdf assumption of Eq. (9), the Maximum Likelihood estimators of $\hat{\mu}$ and $\hat{\theta}$ are deduced in detail. In Table 1 , the estimated parameters $\hat{\mu}$ and $\hat{\theta}$ are shown for different values of the inserted position $L$ and the watermark length $K$. The test images are "Lena" and "Bridge".

Table 1 shows that, as compared with the true scale parameter $\alpha=0.3$, the larger inserted position $L$ and the smaller watermark length $K$ can yield the closer values of $\hat{\alpha}=\hat{\theta} / \hat{\mu}$ to the true value of 0.3 . The reason is that, as shown in Fig. 1(a), the absolute values of $\mathbf{T}$ do not vary acutely for more larger inserted positions $L$. Also, in the shorter lengths $K$ of the watermark $\mathbf{W}$, the absolute values of $\mathbf{T}$ also change slightly. These cases are clearly indicated in Table 1. 
Table 1. Evaluation of the Maximum Likelihood estimators of $\hat{\mu}$ and $\hat{\theta}$ for different values of the position $L$ and the watermark length $K$. The referred images are "Lena" and "Bridge". 1000 different samples of watermarks have been measured, and the true scale parameter $\alpha=0.3$.

\begin{tabular}{|c|c|c|c|c|c|c|}
\hline Image & Inserted position $L$ & Watermark length $K$ & PSNR & $\hat{\mu}$ & $\hat{\theta}$ & $\hat{\alpha}=\hat{\mu} / \hat{\theta}$ \\
\hline \multirow[t]{8}{*}{ Lena } & \multirow[t]{4}{*}{10000} & 3000 & 48.0244 & 31.5119 & 9.6655 & 0.3067 \\
\hline & & 8000 & 44.9235 & 27.3403 & 9.1030 & 0.3330 \\
\hline & & 12000 & 43.9077 & 24.8894 & 8.9288 & 0.3587 \\
\hline & & 20000 & 42.8776 & 21.3211 & 8.8031 & 0.4129 \\
\hline & \multirow[t]{4}{*}{20000} & 3000 & 52.5195 & 18.7955 & 5.6760 & 0.3020 \\
\hline & & 8000 & 49.0038 & 17.19265 & 5.3876 & 0.3134 \\
\hline & & 12000 & 47.7618 & 16.1426 & 5.2524 & 0.3254 \\
\hline & & 20000 & 46.4247 & 14.4587 & 5.1225 & 0.3543 \\
\hline \multirow[t]{8}{*}{ Bridge } & \multirow[t]{4}{*}{10000} & 3000 & 43.7338 & 51.6692 & 15.6199 & 0.3023 \\
\hline & & 8000 & 40.3438 & 46.5113 & 14.7383 & 0.3169 \\
\hline & & 12000 & 39.1560 & 43.3931 & 14.3557 & 0.3308 \\
\hline & & 20000 & 37.8452 & 38.7207 & 13.9295 & 0.3597 \\
\hline & \multirow[t]{4}{*}{20000} & 3000 & 46.9906 & 35.5470 & 10.6570 & 0.2998 \\
\hline & & 8000 & 43.2607 & 33.3666 & 10.1904 & 0.3054 \\
\hline & & 12000 & 41.8769 & 31.8933 & 9.9387 & 0.3116 \\
\hline & & 20000 & 40.3137 & 29.4397 & 9.6245 & 0.3269 \\
\hline
\end{tabular}

Moreover, an example of the histogram of DCT coefficients $\mathbf{T}^{\prime}$ and the corresponding fitted Gaussian mixture pdf $f\left(t^{\prime}\right)$ of Eq. (9), for the inserted position $L=2 \times 10^{4}$ and the watermark length $K=3000$, have been plotted in Fig. 1(c). Clearly, the histogram of $\mathbf{T}^{\prime}$ is separated by the zero-axis to two sets, as shown in Fig. 1(c), each of which collects around its mean values of $\pm \hat{\mu}$, respectively and almost symmetrically. Here, for the generated watermark with seed 100 by MATLAB software, the estimated parameters $\hat{\mu}=18.7801$ and $\hat{\theta}=5.6616$ are computed by Eqs. (A.8) and (A.9) (see Appendix A). It is seen in Fig. 1(c) that this Gaussian mixture pdf model of Eq. (9) presents a good approximation to the statistical histogram of DCT coefficients $\mathbf{T}^{\prime}$.

Furthermore, we also note that the cumulative distribution function (CDF) of Gaussian mixture noise of $\mathbf{T}^{\prime}$ is

$$
F\left(t^{\prime}\right)=\frac{1}{2}\left[\Phi\left(\frac{t^{\prime}-\hat{\mu}}{\hat{\theta}}\right)+\Phi\left(\frac{t^{\prime}+\hat{\mu}}{\hat{\theta}}\right)\right]
$$

where $\Phi(x)=(\sqrt{2 \pi})^{-1} \int_{-\infty}^{x} \exp \left[-z^{2} / 2\right] d z$. For a given significance level 0.1 , we perform a Kolmogorov-Smirnov test [42] to compare the statistical values in the data vector $\mathbf{T}^{\prime}$ with the cumulative distribution function of the Gaussian mixture noise for all values of $L$ and $K$ given in Table 1, and the hypothesis of Eq. (9) can be accepted. An illustrative instance is shown in Fig. 2 for the referred test image "Bridge", where the empirical CDF of marked DCT coefficients $\mathbf{T}^{\prime}$ (dashed line) matches the hypothesized CDF computed by Eq. (10) (solid line) well. Similar results are also tested for other images, e.g., "Lena", "Boat", "Couple" and "Goldhill". 


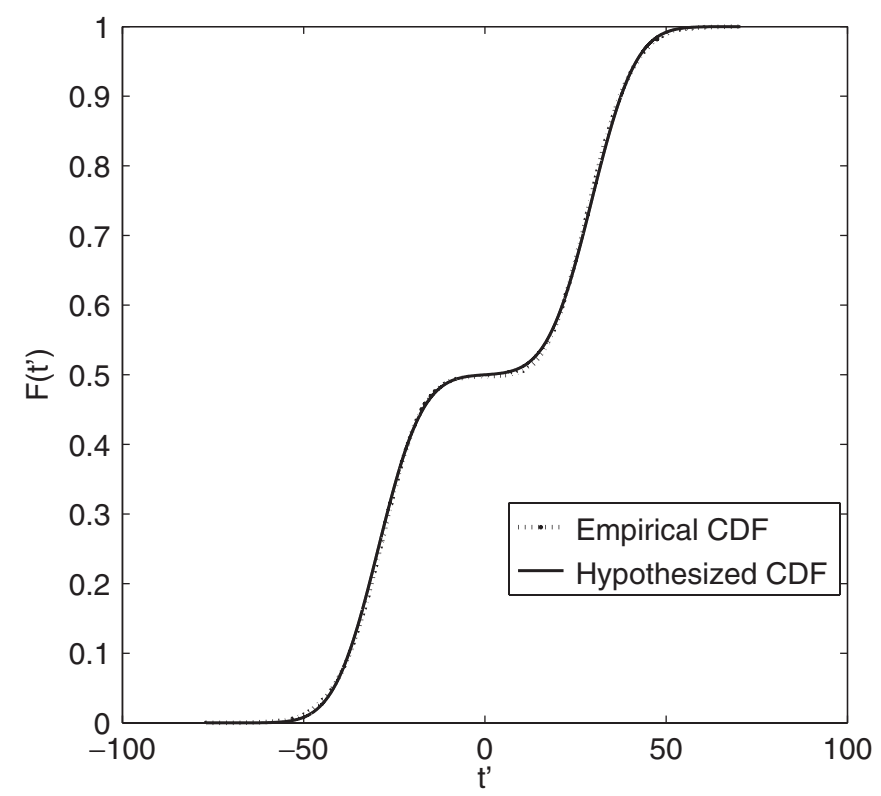

Fig. 2. Empirical CDF of marked DCT coefficients $\mathbf{T}^{\prime}$ (dashed line) and the hypothesized CDF of Eq. (10) (solid line). Here, the marked image is "Bridge". The inserted position $L=20000$, the watermark length $K=20000$ and the scale parameter $\alpha=0.3$. The estimated parameters $\hat{\mu}=29.4428$ and $\hat{\theta}=9.6009$ are computed by Eqs. (A.8) and (A.9) (see Appendix A) for the generated watermark with seed 100 .

Based on the above analyses of pdf models of DCT coefficients $\mathbf{T}$ and marked coefficients $\mathbf{T}^{\prime}$, we approximately demonstrated the assumptions of dichotomous pdf model of Eq. (4) and the Gaussian mixture pdf model of Eq. (9). In this way, the essential watermark embedding rule of Eq. (2) is converted into an additive rule of Eq. (8) that will elicit the following nonlinear detector in next Subsection 2.2.

\subsection{Watermark detector}

Now, according to the additive embedding rule of Eq. (8), the scaled watermark signal $\theta \mathbf{W}$ is submerged in the additive dichotomous noise $\mathbf{T}$ of Eq. (4). It is well known that, from the generalized Neyman-Pearson lemma of detecting a known weak signal in additive non-Gaussian noise, a locally optimum detector [36-41] can be expressed as

$$
\sum_{k=1}^{K}-\theta w_{k} \frac{\frac{d f_{T}\left(t_{L+k}^{\prime}\right)}{d t_{L+k}^{\prime}}}{f_{T}\left(t_{L+k}^{\prime}\right)}>\lambda
$$


for the hypothesis $\mathcal{H}_{1}: \theta>0$, with a decision threshold $\lambda$. Using Eq. (6), Eq. (11) can be expanded as

$$
\lim _{\epsilon \rightarrow 0}\left(\sum_{k=1}^{K} w_{k}\left[t_{L+k}^{\prime}-\mu \tanh \left(\frac{\mu t_{L+k}^{\prime}}{\epsilon^{2}}\right)\right]\right)>\lim _{\epsilon \rightarrow 0}\left(\frac{\epsilon^{2} \lambda}{\theta}\right)
$$

with

$$
\begin{aligned}
\frac{\frac{d f_{T}\left(t_{L+k}^{\prime}\right)}{d t_{L+k}^{\prime}}}{f_{T}\left(t_{L+k}^{\prime}\right)} & =\lim _{\epsilon \rightarrow 0}-\frac{d \kappa\left(t_{L+k}^{\prime} \mid \mu, \epsilon\right)}{d t_{L+k}^{\prime}} \\
& =\lim _{\epsilon \rightarrow 0}\left[-\frac{t_{L+k}^{\prime}}{\epsilon^{2}}+\frac{\mu}{\epsilon^{2}} \tanh \left(\frac{\mu t_{L+k}^{\prime}}{\epsilon^{2}}\right)\right] .
\end{aligned}
$$

Noting $\lim _{\varepsilon \rightarrow 0} \tanh \left(\mu t_{L+k}^{\prime} / \epsilon^{2}\right)=\operatorname{sign}\left(t_{L+k}^{\prime}\right)$, Eq. (12) is then expressed as

$$
\mathbf{D}=\sum_{k=1}^{K} w_{k}\left[t_{L+k}^{\prime}-\mu \operatorname{sign}\left(t_{L+k}^{\prime}\right)\right]>0
$$

where $\operatorname{sign}(\cdot)$ is the sign or signum function. Under the assumptions of a dichotomous noise of Eq. (4), the test statistic D in Eq. (13) being larger than zero indicates that the signal $\mathbf{W}$ is present; that is, the hypothesis $\mathcal{H}_{1}: \theta>0$. However, the more practical task is that the test statistic $\mathbf{D}$ in Eq. (13) can be utilized to determine whether a given watermark is the true one from a set of known watermarks [18]. Concretely, the issue that we are usually confronted with is a pirate claiming another arbitrary watermark $\mathbf{W}^{\prime}=\left\{w_{1}^{\prime}, w_{2}^{\prime}, \ldots, w_{K}^{\prime}\right\}$ being embedded into the given data $\mathbf{T}^{\prime}$. Thus, for a set of known watermark $\mathbf{W}^{\prime}$ s that include the true one $\mathbf{W}$, we are more interested in the following hypotheses test

$$
\begin{array}{ll}
\mathcal{H}_{0}: f\left(t^{\prime}\right) & \text { for } \mathbf{W}^{\prime} \neq \mathbf{W}, \\
\mathcal{H}_{1}: f\left(t^{\prime}\right) & \text { for } \mathbf{W}^{\prime}=\mathbf{W} .
\end{array}
$$

Based on the locally optimum detector of Eq. (13), we compare the test statistic D in Eq. (13) with a decision threshold $\gamma$ for distinguishing the right watermark $\mathbf{W}$ from wrong ones $\mathbf{W}^{\prime}$ s. This is

$$
\mathbf{D}=\sum_{k=1}^{K} w_{k}^{\prime}\left[t_{L+k}^{\prime}-\mu \operatorname{sign}\left(t_{L+k}^{\prime}\right)\right]>\gamma
$$

which is named by the sign detector here. The performance of the sign detector of Eq. (15) will be discussed in detail in Sec. 2.3. Moreover, the threshold $\gamma$ will be solved for a given false alarm probability. 


\subsection{Theoretical analysis of the sign detector of Eq. (15)}

Assume the components $w_{k} \mathrm{~s}$ of the correct watermark $\mathbf{W}$ and $w_{k}^{\prime} \mathrm{s}$ of an arbitrary watermark $\mathbf{W}^{\prime}$ are zero mean, independent and equally distributed normal random variables, and the given data $\mathbf{T}^{\prime}$ contains the signal $\mathbf{W}$ actually. Then, for an arbitrary watermark signal $\mathbf{W}^{\prime}$ that might be the correct one or quoted by the pirate, we substitute $t_{L+k}^{\prime}$ in the transformed embedding rule of Eq. (8) into the test statics $\mathbf{D}$, and obtain

$$
\begin{aligned}
\mathbf{D} & =\sum_{k=1}^{K} w_{k}^{\prime}\left[t_{L+k}^{\prime}-\mu \operatorname{sign}\left(t_{L+k}^{\prime}\right)\right] \\
& =\sum_{k=1}^{K} w_{k}^{\prime}\left[t_{L+k}+\theta w_{k}-\mu \operatorname{sign}\left(t_{L+k}+\theta w_{k}\right)\right] \\
& \approx \sum_{k=1}^{K} \theta w_{k}^{\prime} w_{k}+\sum_{m=1}^{K^{\prime} / 2} 2 \mu w_{m}^{\prime}-\sum_{n=1}^{K^{\prime} / 2} 2 \mu w_{n}^{\prime} .
\end{aligned}
$$

The reason is that, due to the addition of $\theta w_{k}$ to $t_{L+k}$, some $t_{L+k} \mathrm{~s}$ will change their signs. The change of sign indicates the value of $t_{L+k}+\theta w_{k}$ crossing zero. Therefore, using Eq. (9), the number of the changed signs of $t_{L+k} \mathrm{~s}$ are $K^{\prime} / 2 \approx\lceil K Q(\mu / \theta)\rceil / 2$ for both positive and negative values of $t_{L+k} \mathrm{~s}$. Here, $\lceil\cdot\rceil$ finds a next smallest integer and the right-tail probability function $Q(x)=\int_{x}^{\infty} \exp \left[-z^{2} / 2\right] / \sqrt{2 \pi} d z[37,42]$.

From Eq. (16), the mean of $\mathbf{D}$ can be computed as

$$
\mathrm{E}\left[\mathbf{D} ; \mathcal{H}_{0}\right] \approx 0, \quad \mathrm{E}\left[\mathbf{D} ; \mathcal{H}_{1}\right] \approx \theta K
$$

whereby the expectation of $\sum_{k=1}^{K} \theta w_{k}^{\prime} w_{k}$ in Eq. (16) is zero for the statistically independent vectors $\mathbf{W}^{\prime} \neq \mathbf{W}$ of the hypothesis $\mathcal{H}_{0}: \mathbf{W}^{\prime} \neq \mathbf{W}$, while $\sum_{k=1}^{K} \theta w_{k}^{2}$ accord with the $\chi^{2}(K)$ pdf with the degree of freedom $K[37,42]$ for the hypothesis $\mathcal{H}_{1}: \mathbf{W}^{\prime}=\mathbf{W}$ and $\mathrm{E}\left[\sum_{k=1}^{K} \theta w_{k}^{2}\right] \approx \theta K$. For both hypotheses, the expectation of last two terms of Eq. (16) $\mathrm{E}\left[\sum_{m=1}^{K^{\prime}} \mu w_{m}^{\prime}-\sum_{n=1}^{K^{\prime}} \mu w_{n}^{\prime}\right] \approx 0$. Also, according to the above pdf models of the test statistics $\mathbf{D}$ for both hypotheses $\mathcal{H}_{0}$ and $\mathcal{H}_{1}$, the variances of $\mathbf{D}$ can be approximately computed as

$$
\operatorname{Var}\left[\mathbf{D} ; \mathcal{H}_{0}\right] \approx K \theta^{2}+4 K^{\prime} \mu^{2}, \quad \operatorname{Var}\left[\mathbf{D} ; \mathcal{H}_{1}\right] \approx 2 K \theta^{2}+4 K^{\prime} \mu^{2}
$$

For a sufficiently large length $K$, we usually evaluate the asymptotic pdf of the test statistic $\mathbf{D}$ as a Gaussian distribution model with the mean E $[\mathbf{D}]$ and the variance $\operatorname{Var}[\mathbf{D}][37,42]$. Thus, for a given false alarm probability $\mathbf{P}_{\text {FA }}[37,42]$, the decision threshold $\gamma$ can be expressed as

$$
\gamma \approx \sqrt{\operatorname{Var}\left[\mathbf{D} ; \mathcal{H}_{0}\right]} Q^{-1}\left(\mathbf{P}_{\mathbf{F A}}\right)=\sqrt{K \theta^{2}+4 K^{\prime} \mu^{2}} Q^{-1}\left(\mathbf{P}_{\mathbf{F A}}\right)
$$


where $Q^{-1}(x)$ is the inverse function of $Q(x)$. Then, using Eq. (19), the detection probability $\mathbf{P}_{\mathbf{D}}$ can be written as

$$
\begin{aligned}
\mathbf{P}_{\mathbf{D}} & \approx Q\left[\frac{\gamma-\mathrm{E}\left[\mathbf{D} ; \mathcal{H}_{1}\right]}{\sqrt{\operatorname{Var}\left[\mathbf{D} ; \mathcal{H}_{1}\right]}}\right]=Q\left[\frac{\sqrt{K \theta^{2}+4 K^{\prime} \mu^{2}} Q^{-1}\left(\mathbf{P}_{\mathbf{F A}}\right)-\theta K}{\sqrt{2 K \theta^{2}+4 K^{\prime} \mu^{2}}}\right] \\
& \approx Q\left[\frac{\sqrt{1+4\left(\frac{\mu}{\theta}\right)^{2} Q\left(\frac{\mu}{\theta}\right)} Q^{-1}\left(\mathbf{P}_{\mathbf{F A}}\right)-\sqrt{K}}{\sqrt{2+4\left(\frac{\mu}{\theta}\right)^{2} Q\left(\frac{\mu}{\theta}\right)}}\right] \\
& =Q\left[\frac{\sqrt{1+4 \alpha^{-2} Q\left(\alpha^{-1}\right)} Q^{-1}\left(\mathbf{P}_{\mathbf{F A}}\right)-\sqrt{K}}{\sqrt{2+4 \alpha^{-2} Q\left(\alpha^{-1}\right)}}\right],
\end{aligned}
$$

which is the function of the ratio $\mu / \theta=\alpha^{-1}$ and the watermark length $K$. It is noted in Eq. (8) that parameters $\mu$ and $\theta$ actually indicate the RMS values of the noise $\mathbf{T}$ and the signal $\mathbf{W}$, respectively. Thus, the scale parameter $\theta / \mu=\alpha$ can be also regarded as the input signal-to-noise ratio. For a fixed false alarm probability $\mathbf{P}_{\mathbf{F A}}$ and given input signal-to-noise ratio $\alpha$, Eq. (20) indicates that the detection probabilities $\mathbf{P}_{\mathbf{D}}$ is a monotonic increasing function of the watermark length $K$.

\subsection{Evaluation of the sign detector under attacks}

The above theoretical analyses of the sign detector of Eq. (15) are calculated by utilizing the marked DCT coefficients $\mathbf{T}^{\prime}=\left\{t_{L+1}^{\prime}, t_{L+2}^{\prime}, \ldots, t_{L+K}^{\prime}\right\}$. For practical, reasons, for a given data $\mathbf{T}^{\prime}$, the parameters $\mu$ and $\theta$ are unknown for watermark detection, and should be estimated by the Maximum Likelihood method in Appendix A. Moreover, when the marked image has been corrupted by intentional or unintentional attacks, the data that is utilized for estimating $\mu$ and $\theta$ is the corrupted DCT coefficients $\mathbf{T}^{*}=\left\{t_{L+1}^{*}, t_{L+2}^{*}, \ldots, t_{L+K}^{*}\right\}$ taken from the attacked image $\mathbf{I}^{*}$. Throughout the rest of the paper, we still assume the corrupted coefficients $\mathbf{T}^{*}$ with a Gaussian mixture pdf. On the basis of this assumption, for a given data $\mathbf{T}^{*}$, the estimated parameter of $\hat{\mu}$ of Eq. (A.8) and $\hat{\theta}$ of Eq. (A.9) can be computed by substituting $t_{L+k}^{*}$ for $t_{L+k}^{\prime}$. Likewise, the previous expression of the sign detector of Eq. (15) can be rewritten as

$$
\mathbf{D}=\sum_{k=1}^{K} w_{k}^{\prime}\left[t_{L+k}^{*}-\hat{\mu} \operatorname{sign}\left(t_{L+k}^{*}\right)\right]>\gamma,
$$

and, for a given false alarm probability $\mathbf{P}_{\mathbf{F A}}$, the threshold $\gamma$ becomes

$$
\gamma=\sqrt{K \hat{\theta}^{2}+5 K^{\prime} \hat{\mu}^{2}} Q^{-1}\left(\mathbf{P}_{\mathbf{F A}}\right)
$$


with $K^{\prime} \approx\lceil K Q(\hat{\mu} / \hat{\theta})\rceil$. The detection probability $\mathbf{P}_{\mathbf{D}}$ of Eq. (20) can be recalculated as

$$
\mathbf{P}_{\mathbf{D}}=Q\left[\frac{\sqrt{1+4\left(\frac{\hat{\mu}}{\hat{\theta}}\right)^{2} Q\left(\frac{\hat{\mu}}{\hat{\theta}}\right)} Q^{-1}\left(\mathbf{P}_{\mathbf{F A}}\right)-\sqrt{K}}{\sqrt{2+4\left(\frac{\hat{\mu}}{\hat{\theta}}\right)^{2} Q\left(\frac{\hat{\mu}}{\hat{\theta}}\right)}}\right]
$$

with the estimated parameters $\hat{\mu}$ to $\hat{\theta}$ computed by Eqs. (A.8) and (A.9) (see Appendix A). These modified formulae of the sign detector of Eq. (21), the threshold of Eq. (22) and the detection probability of Eq. (23) will be testified by the following robustness experiments in Sec. 3 .

\section{Experimental Results}

In order to verify the effectiveness of the proposed watermark scheme, we test some commonly used $512 \times 512$ gray images with no attack and some common attacks. The following results refer to the standard test images "Lena", "Bridge", "Boat", "Goldhill", and "Couple". According to the analysis of the detection probability $\mathbf{P}_{\mathbf{D}}$ of Eq. (20) above, the larger the watermark length $K$ is, the better performance the detector of Eq. (15) has. Here, we chose a sufficient large length $K=2 \times 10^{4}$ and the scale parameter $\alpha=0.3$.

In view of the invisibility of watermark and the assumption of ordered DCT coefficients as dichotomous noise, we select the position $L=1.5 \times 10^{4}$ to insert the watermark signal in the embedding rule of Eq. (8). For the five test images of "Lena", "Bridge", "Boat", "Goldhill", and "Couple", the corresponding PSNRs are all larger than $39 \mathrm{~dB}$. We note that the position $L$, the watermark length $K$ and the scale parameter $\alpha$ can all affect the PSNR of watermarked image and the detection probability. And it is interesting to study the optimality of the position $L$, the watermark length $K$ and the scale parameter $\alpha$ further. In the following robustness experiments of the watermark against some common attacks, we will fix the false alarm probability $\mathbf{P}_{\mathbf{F A}}=10^{-6}$. And for each attack test, the experiments are carried on $10^{7}$ times for obtaining the actual values of detection probabilities $\mathbf{P}_{\mathbf{D}}$.

\subsection{Experimental results of JPEG coding}

Figure 3(a) shows the detection probability $\mathbf{P}_{\mathbf{D}}$ as a function of the JPEG compression for the five test images. When the JPEG quality factor degrades from $10 \%$ to $1 \%$, as illustrated in Fig. 3(a), the detection probability $\mathbf{P}_{\mathbf{D}}$ of Eq. (23) also decreases gradually. It is noted that, as the JPEG quality factor is not less than $8 \%$, the detection probability $\mathbf{P}_{\mathbf{D}}$ always keeps $100 \%$ for the five test images attacked by JPEG coding. It is noteworthy in Fig. 3(a) that, the detection probability $\mathbf{P}_{\mathbf{D}}$ still keeps $100 \%$ for the test image of "Bridge", even the JPEG quality factor is reduced to $4 \%$. At the JPEG quality factor $8 \%$ of the referred test image "Lena", Fig. 3(b) 


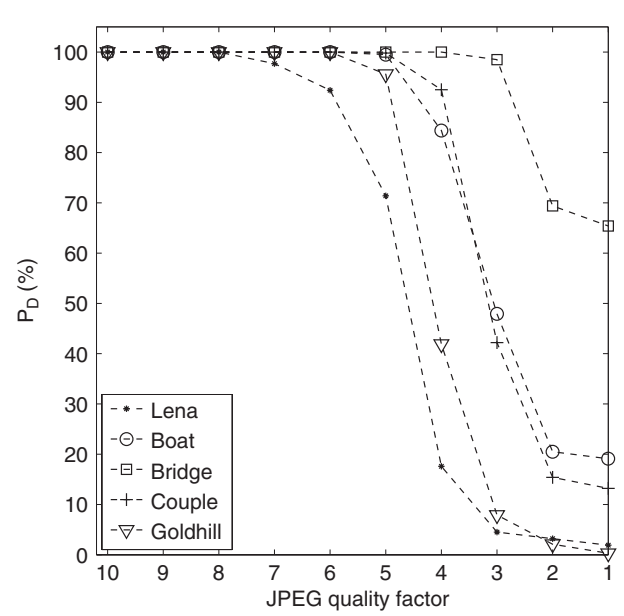

(a)

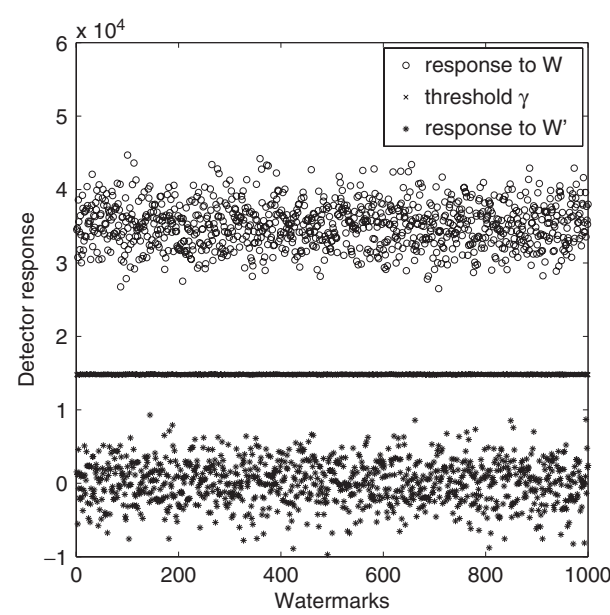

(b)

Fig. 3. Robustness results of watermark against the attack of JPEG coding. (a) The detection probability $\mathbf{P}_{\mathbf{D}}$ as a function of JPEG quality factor for the five test images indicated in the legend. (b) At the JPEG quality factor $8 \%$ of the test image "Lena", detector responses of Eq. (21) to 1000 correct watermarks Ws (o) versus 1000 randomly generated wrong marks W's (*). For each test, the threshold $\gamma(\times)$ of Eq. (22) is also plotted. Here, the watermark length $K=2 \times 10^{4}$, the scale parameter $\alpha=0.3$ and the position $L=1.5 \times 10^{4}$.

illustratively plots the detector responses of Eq. (21) to 1000 correct watermarks Ws versus 1000 randomly generated wrong marks $\mathbf{W}^{\prime}$ s. In addition, the corresponding detection thresholds $\gamma$ of Eq. (22) are depicted in Fig. 3(b), which clearly separate the corresponding responses of detector to $\mathbf{W}$ s and $\mathbf{W}^{\prime}$ s. These experimental results demonstrate the theoretical analyses of Eqs. (21)-(23) well.

\subsection{Experimental results of attacks of median filtering and low pass filtering}

Figure 4(a) describes the test results of the detection probability $\mathbf{P}_{\mathbf{D}}$ versus the median filtering with window sizes of $3 \times 3,5 \times 5,7 \times 7$ and $9 \times 9$. It is seen that, for the median filter with window sizes $3 \times 3$ and $5 \times 5$, the detector of Eq. (21) can correctly detect the embedded watermarks with $\mathbf{P}_{\mathbf{D}}=100 \%$ for the five test images. When the median filter is of window sizes $7 \times 7$ and $9 \times 9$, as shown in Fig. 4(a), the performances of the detector of Eq. (21) are degraded rapidly. We also show the robustness results of watermark against the low pass filtering in Fig. 4(b). The low pass filter is with window sizes of $5 \times 5,6 \times 6$ and $7 \times 7$. The results demonstrate that watermarking is robust to low pass filtering of window size $5 \times 5$ without errors for the five test images. When the window size of the low pass filter is $6 \times 6$, the test images "Boat" and "Bridge" still stand against this kind of filtering attack. 


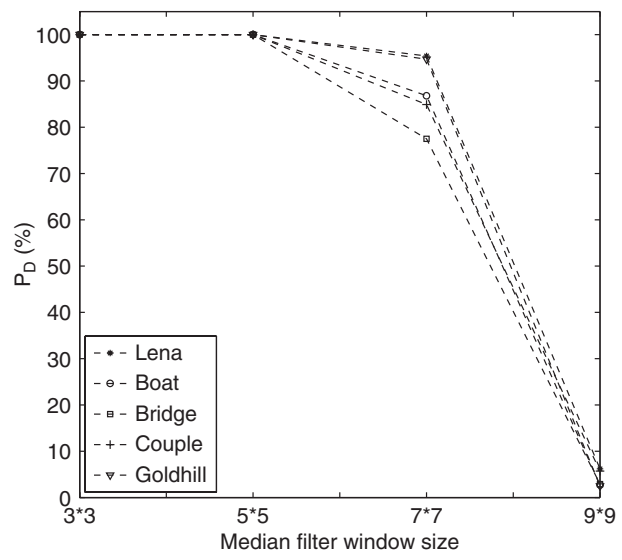

(a)

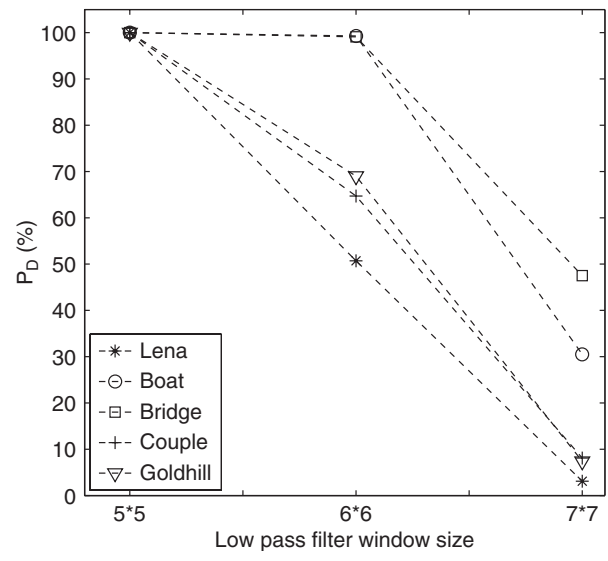

(b)

Fig. 4. Robustness results of watermark against filtering attacks. The detection probability $\mathbf{P}_{\mathbf{D}}$ as a function of (a) the median filtering window size and (b) the low pass filtering window size. The watermark length $K=2 \times 10^{4}$, the scale parameter $\alpha=0.3$ and the position $L=1.5 \times 10^{4}$.

\subsection{Experimental results of additive noise and multiplicative noise attacks}

Figure 5(a) depicts the robustness results of the watermark against the spatial Gaussian noise to corrupt the watermarked image. It is seen that, for the spatial Gaussian noise variance of up to 6400, the nonlinear sign detector of Eq. (21) can

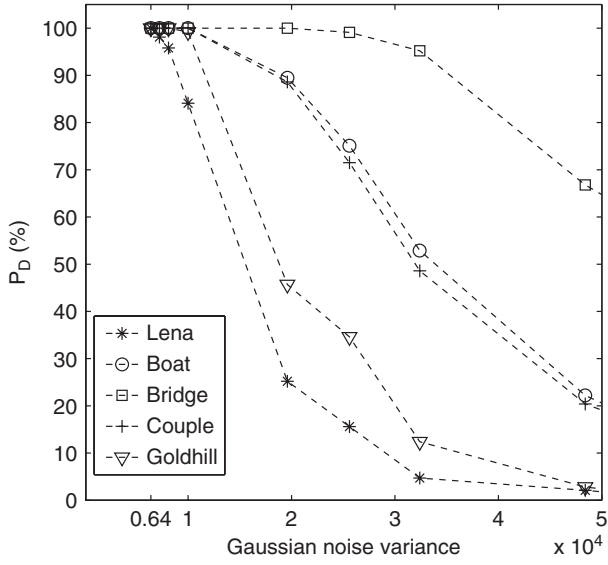

(a)

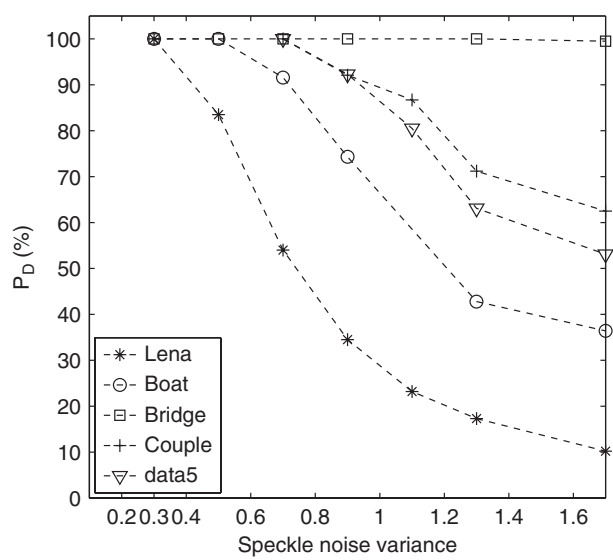

(b)

Fig. 5. Robustness results of watermark against noise corruption. Detection probability $\mathbf{P}_{\mathbf{D}}$ as a function of (a) the additive Gaussian noise variance and (b) the multiplicative speckle noise variance. The watermark length $K=2 \times 10^{4}$, the scale parameter $\alpha=0.3$ and the position $L=1.5 \times 10^{4}$. 
properly detect the right watermarks with $\mathbf{P}_{\mathbf{D}}=100 \%$. As the noise variance is larger than 6400, the detector cannot decipher the correct watermark without errors, and $\mathbf{P}_{\mathbf{D}}$ decreases gradually. In Fig. 5(b), the robustness results of watermarks against the multiplicative speckle noise are illustrated. It is seen that the detector is able to detect the correct watermark perfectly in the presence of the speckle noise variance of less than 0.3 .

\subsection{Experimental results of cropping and rotating}

When a subpart of the watermarked image is cropped, as indicated in Fig. 6(a), the extracted image subpart can be framed via a black background [18]. Then, the cropped image is composed again as a $512 \times 512$ grey one, and the mentioned watermark recovery process can be performed subsequently. However, the black background of supplemented pixel values will bring some very large outliers into the DCT coefficients, as shown in Fig. 6(b). For detecting the watermark, the absolute values of the DCT coefficients beyond 50 are constrained by setting to zero. Here, the value of 50 is an experiential numeral, which can be referred to the marked DCT coefficients of Fig. 1(b). In this way, we test the performance of the detector of Eq. (21), and plot the corresponding results to demonstrate robustness in Fig. 6(c). It is shown in Fig. 6(c) that until the watermarked image is cropped as $12.36 \%$ of the original image, i.e., $180 \times 180$ subpart of the image, the detector of Eq. (21) can detect the right watermark with the detection probability $\mathbf{P}_{\mathbf{D}}=1$ for the five test images.

Figure 7 shows the detection probability $\mathbf{P}_{\mathbf{D}}$ as a function of rotating angle of test images. It is noted that the watermark can survive small rotations up to $0.4^{\circ}$ with the detection probability $\mathbf{P}_{\mathbf{D}}=1$. As for rotating the image with $0.5^{\circ}$, the detection probability $\mathbf{P}_{\mathrm{D}^{\mathrm{S}}}$ are degraded to $87.8 \%, 94.5 \%, 89.6 \%, 78.7 \%$ and $61.5 \%$, for the test images of "Lena", "Boat", "Bridge", "Couple" and "Goldhill", respectively. Note that some parts of the image convey more information than others, and it will be interesting to test the influence of the cropped part of the image on the detection probability $\mathbf{P}_{\mathbf{D}}$.

\subsection{Experimental results of attacks of dithering and desynchronization}

For the dithered versions of the five test images, the corresponding robustness experiments demonstrate that the detector of Eq. (21) can identify the right watermarks embedded in the images with the detection probability $\mathbf{P}_{\mathbf{D}}=1$.

Watermarking desynchronization is one of the most effective attack against any digital watermarking scheme $[1,7,8,10,15,34,35]$. In the proposed watermarking scheme of Sec. 2, the original $K$ positions of the DCT coefficients $\mathbf{T}$ in the host image are viewed as a secret key set for the watermarking detection. For distinguishing right from wrong watermarks, these $K$ positions of $\mathbf{T}$ must be available for the detector of Eq. (21). However, if the detector has difficulty in retrieving these $K$ 


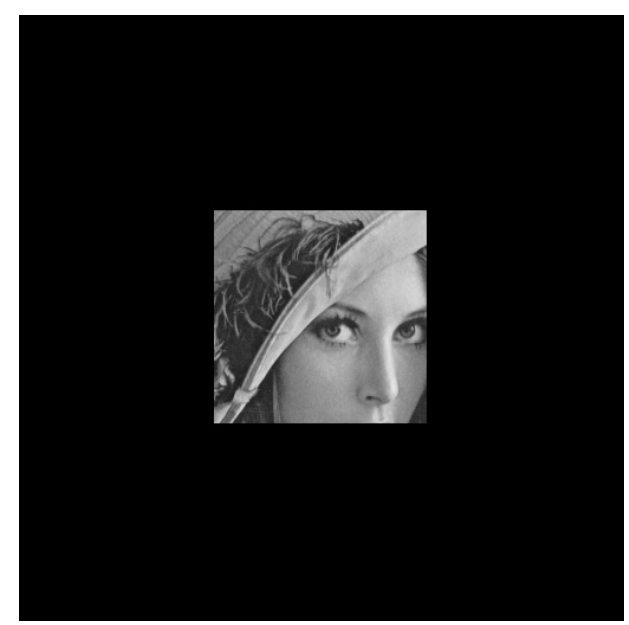

(a)

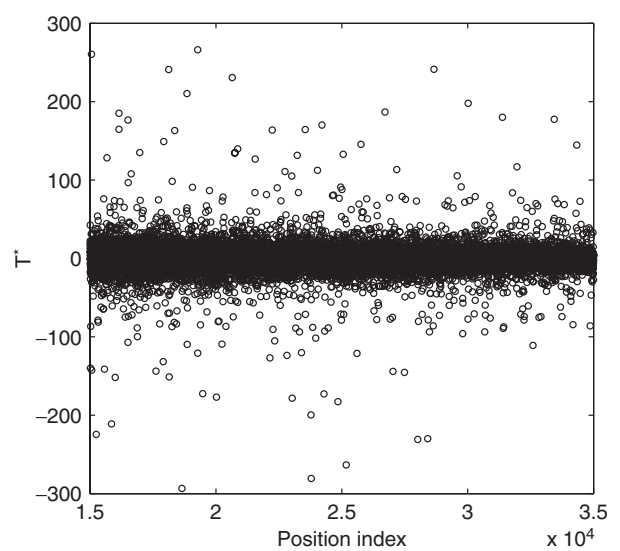

(b)

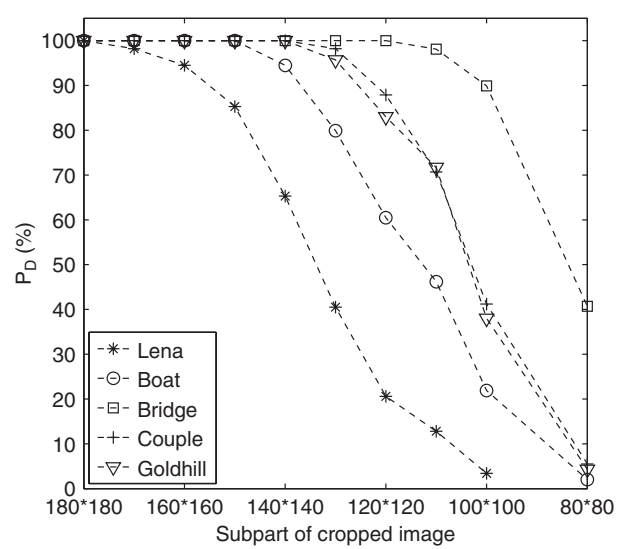

(c)

Fig. 6. Robustness results of watermark against image geometric cropping. (a) The watermarked image "Lena" cropped as $180 \times 180$ with a black background frame. (b) The corresponding marked DCT coefficients $\mathbf{T}^{*}$. (c) The detection probability $\mathbf{P}_{\mathbf{D}}$ versus the subpart area of test images. The watermark length $K=2 \times 10^{4}$, the scale parameter $\alpha=0.3$ and the position $L=1.5 \times 10^{4}$.

positions, the desynchronization between the embedding process and detecting procedure appears, i.e., the desynchronization attack. As shown in Fig. 1(a), the wellordered DCT coefficients $\mathbf{T}$ sorted by their decreased absolute values are disordered randomly by inserting the watermark $\mathbf{W}$ into themselves, resulting in the marked DCT coefficients $\mathbf{T}^{\prime}$ of Fig. 1(b).

When the secret key set of $K$ positions are unknown to the detector, the sorting operation of absolute values cannot recover the order of watermarked coefficients $\mathbf{T}^{\prime}$. For the five test images, the detector of Eq. (21) fails to detect the correct watermark 


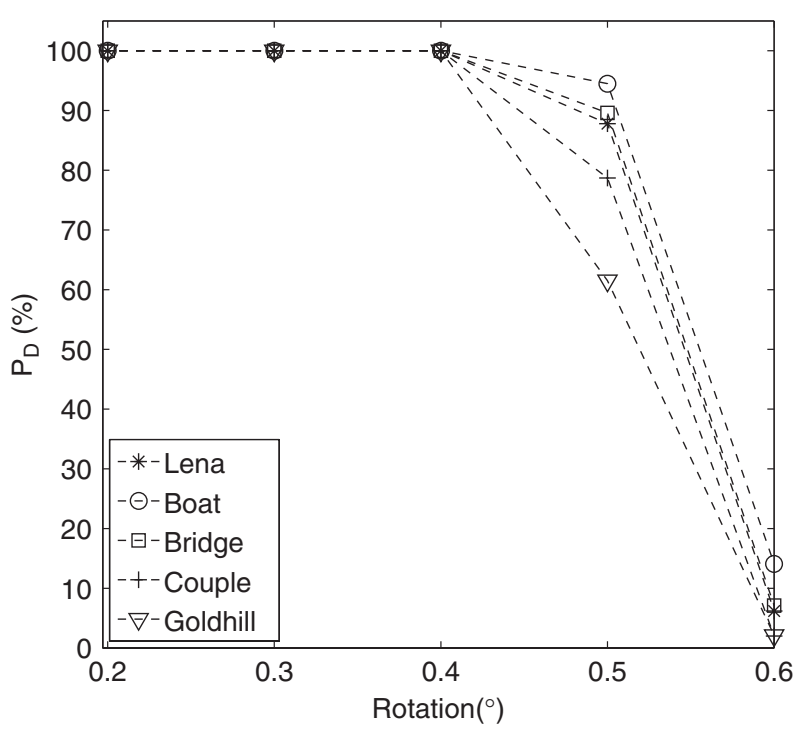

Fig. 7. Robustness result of watermark against image geometric rotating. The detection probability $\mathbf{P}_{\mathbf{D}}$ as a function of rotating angle of test images. The watermark length $K=2 \times 10^{4}$, the scale parameter $\alpha=0.3$ and the position $L=1.5 \times 10^{4}$.

under this kind of desynchronization attack. For the classical watermarking scheme [18], the detector needs to know the watermark $\mathbf{W}$ and the inserted position $L$ for watermark detection. The fixed zig-zag scan can easily yields the marked DCT coefficients for watermark detection. Here, the proposed watermarking detection process requires to know not only the watermark $\mathbf{W}$, but also all marked $(L+1)$ th to $(L+K)$ th DCT coefficients positions. We emphasize that the essential feature of this proposed watermarking scheme is in converting the well-known multiplicative embedding rule of Ref. [18] to an additive embedding rule of Eq. (8) that educes the related locally optimal detector of Eq. (21). According to the abovementioned experiments, its robustness capability against noisy corrupting and cropping are outstanding, as shown in Fig. 5 and Fig. 6, but needs to be further improved to be against the desynchronization attack.

\section{Conclusions}

In this paper, we analyzed in detail the performance of a locally optimal detector for a DCT-domain watermarking scheme. According to the reordering rule of decreased absolute amplitudes, the absolute values of the ordered DCT coefficients are approximately the same constant in a certain length that equals to the length of watermark. Then, we assumed the selected segment of the DCT coefficients that the watermark is embedded into as the dichotomous noise. In this way, the classical multiplicative embedding method of Eq. (2) [18] becomes an additive relationship of the DCT coefficients and the watermark signal. Therefore, we are appropriately 
confronted with a well-known signal detection problem of detecting a known weak signal in the additive non-Gaussian noise.

The marked DCT coefficients by watermark is with a Gaussian mixture pdf, and the unknown pdf parameters can be estimated by the Maximum Likelihood method [37, 42]. Based on the generalized Neyman-Pearson lemma, a locally optimum detector, named the sign detector, has been deduced and employed for the watermark detection. Theoretical analyses of the performance of this nonlinear sign detector were discussed, and extensive robustness experiments have been carried out. The corresponding results demonstrated the effectiveness of the proposed sign detector against some common attacks.

Our analysis in this paper elicits a new kind of correlation-based watermarking scheme, with emphasis on the detectability of a nonlinear sign detector. To improve the proposed watermarking scheme, the future research direction will be devoted to enhance the robustness of watermark against attacks of rotation and desynchronization. Moreover, for the maximum allowable energy under the invisibility constraint, ways to tune the watermark length, the inserted position and the scale parameter into an optimal set is also an interesting research direction for robust watermark detection $[17,29]$. We also note that the comparisons between the proposed detector and classical watermark detectors will be of increased interest for further studies [28, 29].

\section{Acknowledgments}

This work is sponsored by the Taishan Scholar CPSP and the Natural Science Foundation of Shandong Province, China (No. ZR2010FM006). Funding from the Australian Research Council (ARC) is gratefully acknowledged.

\section{Appendix A. Parameter Estimations of $\boldsymbol{\mu}$ and $\boldsymbol{\theta}$ for a Gaussian Mixture pdf Model of Eq. (9)}

For the signal W corrupted by additive noise $\mathbf{T}$ of Eq. (4), the marked DCT coefficients $\mathbf{T}^{\prime}$ is with a Gaussian mixture pdf model of Eq. (9). In practice, the parameters $\mu$ and $\theta$ are unknown for a given data vector $\mathbf{T}^{\prime}$. Here, we use the Maximum Likelihood method [37] to estimate the parameters $\mu$ and $\theta$. To do this, the Maximum Likelihood estimators $\hat{\mu}$ and $\hat{\theta}$ can be obtained from the logarithmic likelihood equation as $[37]$

$$
[\hat{\mu}, \hat{\theta}]=\arg \max _{\mu, \theta} \sum_{k=1}^{K} \ln f\left(t_{L+k}^{\prime}\right),
$$

which yields

$$
\frac{\partial \ln f\left(t_{L+k}^{\prime}\right)}{\partial \mu}=0, \quad \frac{\partial \ln f\left(t_{L+k}^{\prime}\right)}{\partial \theta}=0 .
$$


Subsequently, Eq. (A.2) can be expanded as

$$
\begin{array}{r}
\sum_{k=1}^{K}\left[-\frac{\mu}{\theta^{2}}+\frac{t_{L+k}^{\prime}}{\theta^{2}} \tanh \left(\frac{\mu t_{L+k}^{\prime}}{\theta^{2}}\right)\right]=0, \\
\sum_{k=1}^{K}\left[-\frac{1}{\theta}+\frac{t_{L+k}^{\prime 2}+\mu^{2}}{\theta^{3}}-\frac{2 \mu t_{L+k}^{\prime}}{\theta^{3}} \tanh \left(\frac{\mu t_{L+k}^{\prime}}{\theta^{2}}\right)\right]=0 .
\end{array}
$$

Multiply both sides of Eq. (A.3) by $\theta^{2}$, we have

$$
\mu=\frac{1}{K} \sum_{k=1}^{K}\left[t_{L+k}^{\prime} \tanh \left(\frac{\mu t_{L+k}^{\prime}}{\theta^{2}}\right)\right] .
$$

Substituting Eq. (A.5) into Eq. (A.4) yields

$$
\theta=\sqrt{\frac{1}{K} \sum_{k=1}^{K} t_{L+k}^{\prime 2}-\mu^{2}}
$$

and Eq. (A.5) then reduces to

$$
\mu=\varphi(\mu)=\frac{1}{K} \sum_{k=1}^{K}\left[t_{L+k}^{\prime} \tanh \left(\frac{\mu t_{L+k}^{\prime}}{\frac{1}{K} \sum_{k=1}^{K} t_{L+k}^{\prime 2}-\mu^{2}}\right)\right] .
$$

Equations (A.6)-(A.7) are not the close-form solutions for the parameters $\mu$ and $\theta$. Thus, in the numerical experiments, we set an initial value of $\mu_{0}$ and use the fixed point iteration method

$$
\mu_{n+1}=\varphi\left(\mu_{n}\right)=\frac{1}{K} \sum_{k=1}^{K}\left[t_{L+k}^{\prime} \tanh \left(\frac{\mu_{n} t_{L+k}^{\prime}}{\frac{1}{K} \sum_{k=1}^{K} t_{L+k}^{\prime 2}-\mu_{n}^{2}}\right)\right],
$$

to solve Eq. (A.7) for $n=0,1,2, \ldots$. Since $-1 \leq \tanh (x) \leq 1$ and $-\sum_{k=1}^{K}\left|t_{L+k}^{\prime}\right| / K \leq \varphi(\mu) \leq \sum_{k=1}^{K}\left|t_{L+k}^{\prime}\right| / K$ for $-\sum_{k=1}^{K}\left|t_{L+k}^{\prime}\right| / K \leq \mu \leq$ $\sum_{k=1}^{K}\left|t_{L+k}^{\prime}\right| / K$, the fixed point algorithm converges. There are three fixed points of $\pm \mu_{n+1}$ and zero for the iteration of Eq. (A.8). The fixed point iteration method will bring the final numerical solution $\hat{\mu}=\mu_{n+1}$ when the difference $\left|\mu_{n+1}-\mu_{n}\right|$ is less than a sufficiently small constant such as $10^{-3}$. In numerical experiments, we usually take the initial value of $\mu_{0}>10$ and the positive fixed point $\hat{\mu}=\mu_{n+1}$ will be obtained generally. Then, from Eq. (A.6) and Eq. (A.8), the estimated parameter $\hat{\theta}$ is given by

$$
\hat{\theta}=\sqrt{\frac{1}{K} \sum_{k=1}^{K} t_{L+k}^{\prime 2}-\hat{\mu}^{2}} .
$$

Similarly, we also assume the corrupted marked coefficients $\mathbf{T}^{*}$ with a Gaussian mixture pdf for the common attacks on images. On the basis of this assumption, for a given data $\mathbf{T}^{*}$, the estimated parameter of $\hat{\mu}$ of Eq. (A.8) and $\hat{\theta}$ of Eq. (A.9) can be also computed by substituting $t_{L+k}^{*}$ for $t_{L+k}^{\prime}$. 


\section{References}

[1] I. J. Cox, M. L. Miller and J. A. Bloom, Digital Watermarking (San Mateo, CA: Morgan Kaufmann, 2001), pp. 6-22.

[2] J. F. Delaigle, C. D. Vleeschouwer and B. Macq, Watermarking algorithm based on a human visual model, Signal Processing 66(3) (1998) 319-335.

[3] M. D. Swanson, M. Kobayashi and A. H. Tewfik, Multimedia data embedding and watermarking technologies, Proceeding of the IEEE 86(6) (1998) 1064-1087.

[4] A. Z. Tirkel, C. F. Osborne and T. E. Hall, Image and watermark registration, Signal Processing 66(3) (1998) 373-383.

[5] F. A. P. Petitcolas, R. J. Anderson and M. G. Kuhn, Information hiding - a survey, Proceedings of the IEEE 87(7) (1999) 1062-1078.

[6] J. R. Hernández and F. Pérez-González, Statistical analysis of watermarking schemes for copyright protection of images, Proceedings of the IEEE 87(7) (1999) 1142-1166.

[7] I. J. Cox and M. L. Miller, The first 50 years of electronic watermarking, EURASIP Journal on Applied Signal Processing (2002) 126-132.

[8] F. Cayre, C. Fontaine and T. Furon, Watermarking security: Theory and practice, IEEE Transactions on Signal Processing 53(10) (2005) 3976-3987.

[9] S. Baudry, J. F. Delaigle, B. Sankur, B. Macq and H. Maître, Analyses of error correction strategies for typical communication channels in watermarking, Signal Processing 81(6) (2001) 1239-1250.

[10] F. Perez-Gonzalez, J. R. Hernández and F. Balado, Approaching the capacity limit in image watermarking: A perspective on coding techniques for data hiding application, Signal Processing 81(6) (2001) 1215-1238.

[11] S. Pereira, S. Voloshynoskiy and T. Pun, Optimal transform domain watermark embedding via linear programming, Signal Processing 81(6) (2001) 1251-1260.

[12] J. R. Hernández, J. M. Rodríguez, F. Pérez-González, Improving the performance of spatial watermarking of images using channel coding, Signal Processing 80(7) (2000) $1261-1279$.

[13] N. Nikolaidis and I. Pitas, Robust image watermarking in the spatial domain, Signal Processing 66(3) (1998) 385-403.

[14] F. Duan, D. Abbott and F. Chapeau-Blondeau, The application of saturating detectors to a DCT-domain watermarking scheme, Fluctuation and Noise Letters 8(1) (2008) L49-L65.

[15] X. Wang, J. Wu and P. Niu, A new digital image watermarking algorithm resilient to desynchronization attacks, IEEE Transactions on Information Forensics and Security 2(4) (2007) 655-663.

[16] I. J. Cox, J. Kiliant, T. Leighton and T. Shamoon, Secure spread spectrum watermarking for multimedia, IEEE Transactions on Image Processing 6(12) (1997) 16731687.

[17] M. Barni, F. Bartolini, A. D. Rosa and A. Piva, A new decoder for the optimum recovery of nonadditive watermarks, IEEE Transactions on Image Processing 10(5) (2001) 755-766.

[18] M. Barni, F. Bartolini, V. Cappellini and A. Piva, A DCT-domain system for robust image watermarking, Signal Processing 66(3) (1998) 357-372.

[19] A. Briassouli and M. G. Strintzis, Locally optimum nonlinearities for DCT watermark detection, IEEE Transactions on Image Processing 13(12) (2004) 1604-1617.

[20] A. Briassouli, P. Tsakalides and A. Stouraitis, Hidden messages in heavy-tails: DCTdomain watermark detection using alpha-stable models, IEEE Transactions on Multimedia 7(4) (2005) 700-715. 
[21] M. Barni, F. Bartolini, A. D. Rosa and A. Piva, Capacity of full frame DCT image watermarks, IEEE Transactions on Image Processing 9(8) (2000) 1450-1455.

[22] E. Y. Lam and J. W. Goodman, A mathematical analysis of the DCT coefficient distributions for images, IEEE Transactions on Image Processing 9(10) (2000) 1661-1666.

[23] F. Müller, Distribution shape of two-dimensional DCT coefficients of natural images, Electronics Letters 29(22) (1993) 1935-1936.

[24] R. L. Joshi and T. R. Fischer, Comparison of generalized Gaussian and Laplacian modeling in DCT image coding, IEEE Signal Processing Letters 2(5) (1995) 81-82.

[25] F. Bartolini, M. Barni and A. Piva, Performance analysis of ST-DM watermarking in presence of nonadditive attacks, IEEE Transactions on Signal Processing 52(10) (2004) 2965-2974.

[26] J. R. Hernández, M. Amado and F. Pérez-González, DCT-domain watermarking techniques for still images: Detector performance analysis and a new structure, IEEE Transactions on Image Processing 9(1) (2000) 55-68.

[27] Q. Cheng and T. S. Huang, Robust optimum detection of transform domain multiplicative watermarks, IEEE Transactions on Signal Processing 51(4) (2003) 906-924.

[28] S. Stanković, I. Djurović, R. Herpers and L. Stanković, An approach to optimal watermark detection, International Journal of Electronics and Communications 57(5) (2003) 355-357.

[29] M. Barni, F. Bartolini, A. D. Rosa and A. Piva, Optimum decoding and detection of multiplicative watermarks, IEEE Transactions on Signal Processing 51(4) (2003) $1118-1123$.

[30] T. K. Tsui, X. Zhang and D. Androutsos, Color image watermarking using multidimensional fourier transforms, IEEE Transactions on Information Forensics and Security 3(1) (2008) 16-28.

[31] F. Cayre and B. Macq, Data hiding on 3-D triangle meshes, IEEE Transactions on Signal Processing 51(4) (2003) 939-949.

[32] J. Wang, G. Liu, Y. Dai, J. Sun, Z. Wang and S. Lian, Locally optimum detection for Barni's multiplicative watermarking in DWT domain, Signal Processing 88(1) (2008) 117-130.

[33] H. W. Kim, D. Choi, H. Choi and T. Kim, Selective correlation detector for additive spread spectrum watermarking in transform domain, Signal Processing 90(8) (2010) 2605-2610.

[34] M. Barni, Effectiveness of exhaustive search and template matching against watermark desynchronization, IEEE Signal Processing Letters 2(3) (2007) 297-310.

[35] S. Voloshynovskiy, S. Pereira, T. Pun, J. J. Eggers and J. K. Su, Attacks on digital watermarks: Classification, estimation based attacks, and benchmarks, IEEE Communications Magazine 39(8) (2001) 118-126.

[36] N. H. Lu and B. Eisenstein, Detection of weak signals in non-Gaussian noise, IEEE Transactions on Information Theory 12(2) (2005) 158-161.

[37] S. Kay, Fundamentals of Statistical Signal Processing: Detection Theory (Englewood Cliffs, NJ: Prentice-Hall, 1998) 751-770.

[38] S. A. Kassam, Signal Detection in Non-Gaussian Noise (Springer-Verlag, New York, 1988).

[39] I. Song and S. A. Kassam, Locally optimum detection of signals in a generalized observation model: The known signal case, IEEE Transactions on Information Theory 36(3) (1990) 502-515. 
[40] K. R. Kolodziejski and J. W. Betz, Detection of weak random signals in IID nonGaussian noise, IEEE Transactions on Communications 48(2) (2000) 222-230.

[41] S. Zozor and P. O. Amblard, Stochastic resonance in locally optimal detectors, IEEE Transactions on Signal Processing 51(12) (2003) 3177-3181.

[42] A. Papoulis and S. U. Pillai, Probability, Random Variables and Stochastic Processes McGraw-Hill (2002) 280-293. 